\title{
Emergence of extended-spectrum $\beta$-lactamase (ESBL) and/or carbapenemase producing Enterobacteriaceae (CPE) and their antimicrobial resistance
}

\author{
Koren $\mathrm{J}^{1}$, Hubenakova $\mathrm{Z}^{1}$, Drahovska $\mathrm{H}^{2}$, Ozaee $\mathrm{E}^{2}$, Markuskova $\mathrm{B}^{2}$, Lichvarikova $\mathrm{A}^{2}$ \\ Institute of Microbiology, Faculty of Medicine, Comenius University and University Hospital Bratislava, \\ Bratislava, Slovakia. jan.koren@fmed.uniba.sk
}

\begin{abstract}
OBJECTIVES: We focused on detecting the most frequent resistance mechanisms in selected multidrugresistant (MDR) strains and determining their antimicrobial resistance.

BACKGROUND: MDR pathogens pose urgent public health threat due to limited treatment options, rigorous control measures and significant mortality.

METHODS: We confirmed extended-spectrum $\beta$-lactamase (ESBL) and carbapenemase producing Enterobacteriaceae through guidelines, as well following $\beta$-lactamases: AmpC by cloxacillin, class $A$ carbapenemase with phenylboronic acid, class B metallo- $\beta$-lactamase with ethylenediaminetetraacetic acid. Multilocus sequence typing was used to investigate 20 Escherichia coli strains.

RESULTS: Overall 205 mostly ESBL Escherichia coli demonstrated resistance against amikacin (4.7 \%), tigecycline $(1.2 \%)$, and no resistance to ceftazidime/avibactam, meropenem, nitrofurantoin and fosfomycin. Out of 41 Klebsiella species (spp.), 37 (90.2\%) showed carbapenemase activity, $13(35.1 \%)$ of class A and $24(64.9 \%)$ of class B. Resistance was following: meropenem $66.7 \%$, tigecyclin $10.2 \%$ and colistin $0 \%$. From Enterobacter spp. 21 strains, 14 (66.7 \%) were ESBL, 5 produced ESBL and/or AmpC and 2 were MDR. We ascertained $14(70 \%)$ E. coli sequence type - ST131.

CONCLUSIONS: The study revealed various resistance mechanisms in concert with different agents and association of specific ST131 within E. coli. These characteristics considerably contribute to emergence of antimicrobial resistance (Tab. 4, Ref. 30). Text in PDF www.elis.sk.

KEY WORDS: ESBL, Escherichia coli, ST131, multidrug-resistance, carbapenemase producing

Enterobacteriaceae.
\end{abstract}

\section{Introduction}

Extended-spectrum $\beta$-lactamase (ESBL) and carbapenemase producing Enterobacterales/Enterobacteriaceae (CPE) pose the highest priority in combating global antibiotic resistance. Their steadily rise with escalating frequency account for concern in healthcare system (1). Carriage and infections caused by ESBL and CPE strains might be the result of inadequate empiric therapy related with mortality of critical ill patients (2). ESBL producing pathogens i.e. Escherichia coli, Klebsiella species (spp.) or Enterobacter spp. are mostly co-resistant to $\beta$-lactams (penicillins, cephalosporins and monobactams) including non- $\beta$-lactam antimicrobial agents - ami-

${ }^{1}$ Institute of Microbiology, Faculty of Medicine, Comenius University and University Hospital Bratislava, Bratislava, Slovakia, and ${ }^{2}$ Department of Molecular Biology, Faculty of Natural Sciences, Comenius University Bratislava, Bratislava, Slovakia

Address for correspondence: J. Koreň, $\mathrm{MD}, \mathrm{PhD}$, Institute of Microbiology, Faculty of Medicine, Comenius University and University Hospital Bratislava, Sasinkova 4, SK-811 08 Bratislava, Slovakia. Phone: $+421.904574270$

Acknowledgements: The study was supported by the project APVV-160173 Ministry of School of the Slovakia. noglycosides, fluoroquinolones, tetracyclines and trimethoprimsulfamethoxazole (3). If aforementioned microorganisms produce AmpC $\beta$-lactamase they are resistant to the above $\beta$-lactams (except 4th generation of cephalosporins), but include cephamycins and inhibitors of $\beta$-lactamase (clavulanic acid, sulbactam and tazobactam) (4). Accordingly, they are multidrug-resistant (MDR; resistance to $\geq 3$ classes of antibiotics) (5). Carbapenems are generally considered as the drugs of choice for the treatment of ESBL and/or AmpC pathogens causing infections, while the other $\beta$-lactams and non- $\beta$-lactams are in vitro efficacious as well. This injudicious carbapenems overuse is giving rise to unpredictable emergence of carbapenem resistant Enterobacteriaceae (CRE) (6). Carbapenemase producing Klebsiella spp. strains are actually extensively drug-resistant (XDR; sensitive to $\leq 2$ classes of antibiotics) (7) etiological agents with high requirement for infection control measures, limited antimicrobial treatment options associated with significant morbidity and mortality rates $(8,9)$. Currently molecular typing-multilocus sequence typing (MLST) is referred as an important approach in infection control that facilitates the detection of nosocomial transmission of pathogens as well as enables prove of source and route of spreading in epidemiological nosocomial environment (10). 


\section{5-940}

The aim of our study was to detect the most frequent mechanisms of resistance in selected ESBL and/or AmpC and CPE strains as well as to evaluate the antimicrobial resistance rate in these investigated pathogens.

\section{Materials and methods}

\section{Specimens and isolates}

Overall biological samples were consecutively recovered from different body sites of patients hospitalized in University Hospital Bratislava (UHB) - Old Town and Specialized geriatric hospital from January 2017 through May 2019. Further examination was performed in our Institute of Microbiology, Faculty of Medicine, Comenius University and UHB. Following primary cultivation and isolation, identification of the strains was carried out biochemically onto: Escherichia coli, Klebsiella pneumoniae and Klebsiella oxytoca (Klebsiella spp.) or Enterobacter cloacae and Enterobacter aerogenes (Enterobacter spp.). Only one non-repetitive isolate per patient was included in the study.

\section{Phenotypic proof of resistance mechanisms}

Concerning primary cultivation, samples were inoculated on chromogenic ESBL culture medium (11) and after 18 24 hours grown strains underwent further confirmatory tests. ESBL - combination disk diffusion test with cefotaxime (30 $\mu \mathrm{g})$ and ceftazidime $(30 \mu \mathrm{g}) \pm$ clavulanic acid $(10 \mu \mathrm{g})$ proved ESBL production if at least in one of $3 \mathrm{rd}$ generation cephalospo- rin $(3 \mathrm{GC})$ in the presence of $\beta$-lactamase inhibitor, the size of inhibition zone (IZ) was $\geq 5 \mathrm{~mm}$ greater compared to IZ of $3 \mathrm{GC}$ alone (12). Evidence of AmpC $\beta$-lactamase was carried out by cefoxitin $(30 \mu \mathrm{g})$ disk $\pm 200 \mu \mathrm{g}$ cloxacillin as inhibitor of AmpC $\beta$-lactamase. Positivity was demonstrated in IZ size of $\geq 4 \mathrm{~mm}$ greater for the disk with added inhibitor than for the cefoxitin disk alone (13). Carbapenemase activity confirmation was provided by chromogenic $\mathrm{pH}$ metric Carba NP test discovered by Nordman et al (2012) (14). In order to achieve precise phenotypic detection of carbapenemase classes in accordance with Ambler, we used two inhibitors: family of serine class A (most common in Slovak republic is KPC - Klebsiella pneumoniae carbapenemase) was determined by meropenem $(10 \mu \mathrm{g}) \pm 400 \mu \mathrm{g}$ phenylboronic acid, and assessment of the zinc-dependent class B, so called metallo$\beta$-lactamase (MBL; most frequent in our country is NDM - New Delhi metallo- $\beta$-lactamase) was achieved by meropenem $(10 \mu \mathrm{g})$ $\pm 292 \mu$ g ethylenediaminetetraacetic acid (EDTA) $(1,12,15)$. In fact, positive CPE strains are sent to National reference center for surveillance of antimicrobial resistance of Slovak republic due to molecular typing.

\section{Genotypic proof of sequence types}

We randomly chose 20 ESBL producing Escherichia coli strains for the (seven-locus) multilocus sequence typing (MLST), including subtyping or two-locus typing method (C-H typing) according to the alleles fum $C$ (fumarate hydratase $C$ ) and fimH (internal fragment of the type 1 fimbrial adhesion $H)$ - encoding genes $(16,17)$.

Tab. 1. Characteristics of patients, healthcare setting and specimens.

\begin{tabular}{|c|c|c|c|c|c|c|}
\hline & \multicolumn{2}{|c|}{ Escherichia coli n 205} & \multicolumn{2}{|c|}{ Klebsiella spp. n 41} & \multicolumn{2}{|c|}{ Enterobacter spp. n 21} \\
\hline & $\mathrm{n}$ & $\%$ & $\mathrm{n}$ & $\%$ & $\mathrm{n}$ & $\%$ \\
\hline \multicolumn{7}{|l|}{ Gender } \\
\hline male & 68 & 33.2 & 17 & 41.5 & 11 & 52.4 \\
\hline female & 137 & 66.8 & 24 & 58.5 & 10 & 47.6 \\
\hline \multicolumn{7}{|l|}{ Age (years) } \\
\hline$<60$ & 12 & 5.9 & 4 & 9.8 & 1 & 4.8 \\
\hline$\geq 60$ & 193 & 94.1 & 37 & 90.2 & 20 & 95.2 \\
\hline mean (range) & 78.9 & $(9-98)$ & 74.4 & $(25-96)$ & 74.9 & $(58-96)$ \\
\hline \multicolumn{7}{|l|}{ Setting } \\
\hline 1st internal clinic & 113 & 55.1 & 23 & 56.1 & 10 & 47.6 \\
\hline Surgical clinic & 14 & 6.8 & 2 & 4.9 & 3 & 14.3 \\
\hline Dermatovenerological clinic & 4 & 2 & 2 & 4.9 & 2 & 9.5 \\
\hline Neurological clinic & 4 & 2 & 1 & 2.4 & - & - \\
\hline Psychiatric clinic & 3 & 1.5 & - & - & - & - \\
\hline Geriatric clinic & 45 & 21.9 & 5 & 12.2 & 3 & 14.3 \\
\hline Aftercare department & 5 & 2.4 & 2 & 4.9 & 2 & 9.5 \\
\hline Outpatient offices & 6 & 2.9 & - & - & - & - \\
\hline Institute of Pathological anatomy & 2 & 1 & 3 & 7.3 & - & - \\
\hline \multicolumn{7}{|l|}{ Type of specimen } \\
\hline rectal swab or stool & 3 & 1.5 & 23 & 56.1 & - & - \\
\hline hemoculture & 15 & 7.3 & - & - & - & - \\
\hline wound, absces or organ swab & 28 & 13.7 & 5 & 12.2 & 5 & 23.8 \\
\hline urine & 140 & 68.3 & 8 & 19.5 & 5 & 23.8 \\
\hline tonsillar swab or sputum & 13 & 6.3 & 5 & 12.2 & 10 & 47.6 \\
\hline catheter related sample & 6 & 2.9 & - & - & 1 & 4.8 \\
\hline
\end{tabular}

$\mathrm{n}$ - number of patients, cases or specimens 
Antibiotic susceptibility testing

Respecting the criteria of European Committee on Antimicrobial Susceptibility Testing (EUCAST) we performed colorimetric micro-method for automated susceptibility testing to determine sensitivity of Escherichia coli, Klebsiella spp. and Enterobacter spp. against 16 antimicrobial agents (18). Additional examination of susceptibility testing strains was possible only by means of available antimicrobial agents and disk diffusion methods as well as consistent with EUCAST breakpoint interpretation of zone diameter (19).

\section{Results}

Isolates, patients, setting and specimens

A total of 205 Escherichia coli, 41 Klebsiella spp. (K. pneumoniae 39 and K. oxytoca 2) as well as 21 Enterobacter spp. (E. cloacae 13, E. aerogenes 5 and Enterobacter spp. 3 - not determined) strains were isolated and included in this survey.

Relating to the patients, mostly strains were isolated from females in the cases of Escherichia coli and Klebsiella spp., but similarly from both genders for Enterobacter spp. (data are depicted in table 1.).

Out of all (from 90.2 - $95.2 \%$ ) were the individuals over 60 years with mean age from $74.4-78.9 \%$ years old. In terms of the hospital setting, the samples were collected from the patients hospitalized particularly at 1st internal clinic (from 47.6 $-56.1 \%$ ), then on Geriatric clinic (from $12.2-21.9 \%$ ), Surgical clinic (from $4.9-14.3 \%$ ), Dermatovenerological clinic (from 2.0 $-9.5 \%$ ), Long-term care department (from $4.4-7.3 \%$ ), Aftercare department (from $2.4-9.5 \%$ ). Few samples (5) were taken into consideration from the patients post mortem from the Institute of Pathological anatomy (expressed in table 1.).
Considering the type of specimen, Escherichia coli was isolated mainly from the 140 (68.3\%) urine samples, then from the skin and soft tissues infections (wounds, abscesses, visceral organs) 28 times (13.7\%), 15 (7.3\%) hemocultures, 13 (6.3\%) samples from tonsillar swabs or sputum and so on. Klebsiella spp 23 (56.1 $\%)$ strains resulted from faecal carriage, $8(19.5 \%)$ were isolated from urine, and for 5 times (12.2\%) from soft tissue infections, as well as from upper and lower respiratory tract. Enterobacter spp. originated from almost half of the samples - 10 (47.6\%) from respiratory tract, quarter samples $-5(23.8 \%)$ were from urine and the equal number from the skin and soft tissue infections, and last one was related to a catheter (but not mentioned which kind), illustrated in Table 1.

\section{Resistance mechanisms}

Evidence of ESBL in Escherichia coli strain was the highest. Out of the total of 205 pathogens, 202 (98.5\%) were ESBL positive and other are presented in table 2. Majority of Klebsiella spp. strains were carbapenemase producers, 37 strains (90.2 \%). From these carbapenemase producing, 24 (64.9\%) were assigned to the metallo- $\beta$-lactamase, Ambler class B and 13 (35.1\%) belonged to the serine class A carbapenemase. Rest of 41 Klebsiella spp. strains were $2(4.9 \%)$ ESBL strains and $2(4.9 \%)$ ESBL/AmpC producers. Enterobacter spp. pathogens were particularly ESBL positive isolates, except for 2 times (9.5\%) MDR strains, as well ESBL/ AmpC combined producers, and 3 (14.3\%) only AmpC producing strains (Tab. 2).

\section{Molecular sequence typing}

MLST of randomly chosen 20 ESBL Escherichia coli strains showed that sequence type - ST131 and C-H type 40 - 30 was detected in $14(70 \%)$ investigated strains. Escherichia coli ST131

Tab. 2. Phenotypically detected resistance mechanisms in Enterobacterales/Enterobacteriaceae strains.

\begin{tabular}{|c|c|c|c|c|c|c|c|c|c|c|c|c|c|}
\hline \multirow{2}{*}{$\begin{array}{l}\text { Etiological } \\
\text { agents }\end{array}$} & \multirow{2}{*}{$\begin{array}{l}\text { total } n \\
\text { of strains }\end{array}$} & \multicolumn{2}{|c|}{ MDR } & \multicolumn{2}{|c|}{ ESBL } & \multicolumn{2}{|c|}{$\mathrm{ESBL}+\mathrm{AmpC}$} & \multicolumn{2}{|c|}{ AmpC } & \multicolumn{2}{|c|}{$\begin{array}{c}\text { ESBL + } \\
\text { carbapenemase }\end{array}$} & \multicolumn{2}{|c|}{ carbapenemase } \\
\hline & & $n$ & $\%$ & $n$ & $\%$ & $n$ & $\%$ & $n$ & $\%$ & $n$ & $\%$ & $n$ & $\%$ \\
\hline Escherichia coli & 205 & - & - & 202 & 98.5 & 2 & 1 & 1 & 0.5 & - & - & - & - \\
\hline Klebsiella spp. & 41 & - & - & 2 & 4.9 & 2 & 4.9 & - & - & 34 & 82.9 & 3 & 7.3 \\
\hline Enterobacter spp. & 21 & 2 & 9.5 & 14 & 66.7 & 2 & 9.5 & 3 & 14.3 & - & - & - & - \\
\hline
\end{tabular}

$n$ - number of evaluated strains; \% - percentage of strains; MDR - multidrug-resistance - resistance to $\geq 3$ drugs from different antimicrobial classes; ESBL - extended-spectrum $\beta$-lactamase; AmpC - type of $\beta$-lactamase

Tab. 3. MLST and C-H typing of randomly chosen 20 ESBL Escherichia coli strains.

\begin{tabular}{|c|c|c|c|c|c|c|c|c|c|c|c|c|}
\hline \multirow[b]{2}{*}{ ST } & \multirow[b]{2}{*}{$n$} & \multirow[b]{2}{*}{$\%$} & \multicolumn{2}{|c|}{ gender } & \multirow[b]{2}{*}{ C-H type } & \multicolumn{3}{|c|}{ specimen } & \multicolumn{4}{|c|}{ setting } \\
\hline & & & male & female & & $\begin{array}{c}\text { urine or } \\
\text { catheter } n\end{array}$ & $\begin{array}{c}\text { skin and } \\
\text { soft tissues } n\end{array}$ & $\begin{array}{l}\text { respiratory } \\
\text { tract } n\end{array}$ & $\begin{array}{l}\text { 1st internal } \\
\text { clinic }\end{array}$ & $\begin{array}{l}\text { Surgical } \\
\text { clinic }\end{array}$ & $\begin{array}{l}\text { Neurological } \\
\text { clinic }\end{array}$ & $\begin{array}{c}\text { Outpatient } \\
\text { office }\end{array}$ \\
\hline 73 & 1 & 5 & - & 1 & $24-184$ & 1 & - & - & 1 & - & - & - \\
\hline 131 & 14 & 70 & 3 & 11 & $40-30$ & $8+1$ & 3 & 2 & 12 & 1 & 1 & - \\
\hline 162 & 1 & 5 & 1 & - & $65-35$ & 1 & - & - & 1 & - & - & - \\
\hline 405 & 1 & 5 & - & 1 & $37-27$ & 1 & - & - & 1 & - & - & - \\
\hline 648 & 1 & 5 & 1 & - & 4-0 & 1 & - & - & 1 & - & - & - \\
\hline 1193 & 1 & 5 & 1 & - & $14-64$ & 1 & - & - & - & - & - & 1 \\
\hline
\end{tabular}

MLST - multilocus sequence typing; C-H typing - according to fumC [fumarate hydratase C] and fimH [fimbrial adhesin $\mathrm{H}$ ]; ESBL - extended-spectrum $\beta$-lactamase; $\mathrm{ST}$ - sequence type; $n$ - number of isolates 
Tab. 4. Antimicrobial resistance of Escherichia coli, Klebsiella spp. and Enterobacter spp. strains.

\begin{tabular}{|c|c|c|c|c|c|c|c|c|c|}
\hline \multirow[b]{2}{*}{ antibiotics } & \multicolumn{3}{|c|}{ Escherichia coli } & \multicolumn{3}{|c|}{ Klebsiella spp. } & \multicolumn{3}{|c|}{ Enterobacter spp. } \\
\hline & $\begin{array}{l}\text { overall } n \text { of } \\
\text { Escherichia } \\
\text { coli }\end{array}$ & $\begin{array}{c}n \text { of } \\
\text { resistant } \\
\text { isolates }\end{array}$ & $\%$ & $\begin{array}{l}\text { overall } n \text { of } \\
\text { Klebsiella } \\
\text { spp. }\end{array}$ & $\begin{array}{l}n \text { of } \\
\text { resistant } \\
\text { isolates }\end{array}$ & $\%$ & $\begin{array}{l}\text { overall } n \text { of } \\
\text { Enterobacter } \\
\text { spp. }\end{array}$ & $\begin{array}{l}\quad n \text { of } \\
\text { resistant } \\
\text { isolates }\end{array}$ & $\%$ \\
\hline ampicillin/sulbactam & 194 & 151 & 77.8 & 39 & 39 & 100 & 10 & 10 & 100 \\
\hline piperacilin/tazobactam & 190 & 33 & 17.4 & 39 & 39 & 100 & 18 & 12 & 66.7 \\
\hline cefuroxime & 193 & 189 & 97.9 & 39 & 39 & 100 & 18 & 17 & 94.4 \\
\hline ceftazidime & 193 & 151 & 78.2 & 39 & 39 & 100 & 18 & 16 & 88.9 \\
\hline ceftazidime/avibactam & 177 & 0 & 0 & 41 & 24 & 58.5 & 19 & 0 & 0 \\
\hline cefoperazone/sulbactam & 166 & 6 & 3.6 & 39 & 35 & 89.7 & 10 & 5 & 50 \\
\hline cefepime & 166 & 136 & 81.9 & 39 & 39 & 100 & 10 & 6 & 60 \\
\hline ceftolozane/tazobactam & 177 & 4 & 2.3 & 41 & 40 & 97.6 & 19 & 2 & 10.5 \\
\hline ertapenem & 166 & 0 & 0 & 39 & 36 & 92.3 & 10 & 0 & 0 \\
\hline gentamicin & 192 & 50 & 26 & 39 & 34 & 87.2 & 18 & 10 & 55.5 \\
\hline tobramycin & 166 & 96 & 57.8 & 39 & 38 & 97.4 & 10 & 6 & 60 \\
\hline amikacin & 193 & 9 & 4.7 & 39 & 29 & 74.4 & 18 & 0 & 0 \\
\hline ciprofloxacin & 194 & 182 & 93.8 & 39 & 39 & 100 & 18 & 11 & 61.1 \\
\hline tigecycline & 166 & 2 & 1.2 & 39 & 4 & 10.2 & - & - & - \\
\hline trimethoprim/sulfamethoxazole & 192 & 146 & 76.0 & 39 & 31 & 79.5 & 18 & 9 & 50 \\
\hline colistin & 164 & 0 & 0 & 38 & 0 & 0 & 10 & 0 & 0 \\
\hline temocillin & 177 & 1 & 0.6 & 32 & 4 & 12.5 & 14 & 0 & 0 \\
\hline nitrofurantoin & 15 & 0 & 0 & - & - & - & - & - & - \\
\hline fosfomycin & 15 & 0 & 0 & - & - & - & - & - & - \\
\hline
\end{tabular}

$n$ - number of strains; $\%$ - percentage of resistance

was isolated in urine specimen 8 times and from urinary catheter once, from skin and soft tissues 3 times and twice from respiratory tract. According to hospital setting this most frequent ST131 had source on 1st internal clinic 12 times (mainly in females) and for once on Surgical and Neurological clinic. Remainder of the sporadic sequence types or C-H types of ESBL Escherichia coli had origin also on 1st internal clinic and other healthcare providing facilities (Tab. 3).

\section{Antibiotic resistance}

Resistance rate in ESBL Escherichia coli evaluated strains was highest against unprotected cephalosporins including fluoroquinolone ciprofloxacin, followed by protected ampicillin/sulbactam and trimethoprim/sulfamethoxazole (Tab. 4). The highest prevalence of resistance to aminoglycosides was against tobramycin and the lowest to amikacin. Lower resistance rate was registered also against cefoperazone/sulbactam, ceftolozane/tazobactam, tigecycline and temocillin. No recorded resistance in Escherichia coli ESBL producing strains against: ceftazidime/avibactam, ertapenem, meropenem, colistin, nitrofurantoin and fosfomycin. Most of the times, in Klebsiella spp. mainly carbapenemase producing strains resistance toward penicillins and unprotected cephalosporins including ciprofloxacin was $100 \%$. Against cephalosporins with inhibitor of $\beta$-lactamase, the lowest resistance rate was to ceftazidime/avibactam. Actually, 13 Klebsiella spp. strains from serine class A carbapenemase (probably KPC) were completely sensitive to ceftazidime/avibactam ( $0 \%$ resistance), but from class
B metallo- $\beta$-lactamase 24 strains were fully resistant (100\% resistance) to this drug (data not shown). Resistance to meropenem and amikacin was lower, but the lowest was recorded to colistin, tigecycline and temocillin. In Enterobacter spp. ESBL producing strains, antibiotic resistance was of the highest degree against $\beta$-lactams and ciprofloxacin. Exception was ceftazidime/avibactam and ceftolozane/tazobactam, to them resistance was not marked or detected up to $10.5 \%$, respectively. Amikacin, meropenem, colistin and temocillin resistance was not demonstrated in ESBL Enterobacter spp. strains (Tab. 4).

\section{Discussion}

In an attempt to investigate occurrence of ESBL and CPE including their resistance for the recent years, we found out that certain pathogens from normal flora might be more related to the gender, age, as well as to healthcare setting, colonization and diseases (or comorbidities). These pathogens are mostly associated with many other risk factors for the transmission and acquisition of MDR strains (2). Authors Cano et al (2018) published lower median age (59 years) as well as lower incidence of male gender $(33.3 \%)$ with carbapenemase producing Klebsiella pneumoniae in their study compared to our findings. As opposed to our results, in this Spanish survey one half of the patients had a surgery intervention with hospitalization at intensive care unit and also bacteraemia (8). In accordance with our records was public data of Han et al (2017) for the median age (73 years) of the patients with 
carbapenem resistant Klebsiella pneumoniae, but the proportion of female gender (50.7\%) was slightly lower. In this American study related to the patients from long-term acute care hospitals, carbapenem resistant $K$. pneumoniae strains were mostly isolated from respiratory source $(59.2 \%)$, urinary tract (37\%) and from blood (9.4\%) (20). That was different from our types of specimens from where we isolated carbapenemase producing Klebsiella spp. Strains, mainly from gastrointestinal tract and less from other locations (table 1.). Barbier et al (20016) and Lafolie et al (2014) presented results of slightly lower levels of Escherichia coli ESBL production in urine $(60 \%)(2,21)$ and in agreement with our data in hemoculture (8\%) (21). In this French study $17.2 \%$ prevalence of ST131 of 261 ESBL E. coli strains was found (21). In our much lower number of evaluated strains, 14 (70\%) were determined as ST131. In addition, C-H typing detected ST131 clonal subgroup $\mathrm{H} 30$ that is responsible in an important way for the fluoroquinolone and MDR E. coli infections around the world (17).

The most common resistance mechanism in E. coli strains is production of ESBL and occasionally can be combined with AmpC mostly plasmid mediated $\beta$-lactamase production. Chromosomal AmpC hyperproduction due to mutation on promotor of $a m p C$ gene is very rare in E. coli strains. ESBL and/or AmpC hyperproduction combined with porin defect - impermeability can lead to the carbapenem resistance $(9,22)$. However, in E. coli we did not detect this carbapenem resistance, not even carbapenemase production in our results. Mechanisms of resistance in Klebsiella spp. are based on ESBL and/or AmpC production and we had 4 strains (Tab. 2) and 1 of them was carbapenem (meropenem) resistant. Remaining 37 Klebsiella spp. strains were carbapenemase producing (34 out of them with ESBL) and 25 (67.6\%) were carbapenem (meropenem) resistant Klebsiella spp. strains. In published results of Lesho et al (2016) CRE or carbapenem (imipenem) resistant Enterobacteriaceae strains were isolated mainly as Klebsiella spp. (113 times) and less frequently in E. coli (21 times) and Enterobacter spp. (10 times). These CRE data of the same authors were even 73-fold lower than carbapenem resistance in Pseudomonas aeruginosa and 210-fold lower than in Acinetobacter spp. strains, where carbapenem resistance is much more frequent (23). Several years ago (2015 - 2016 years) we proved 3 strains of Enterobacter spp. with carbapenemase production but not in this survey. Recently, resistance mechanism of Enterobacter spp. strains is due to ESBL and/or AmpC production. Majority of the strains were ESBL positive (Tab. 2). In Enterobacter spp. is intrinsically present chromosomal encoded class $\mathrm{C}$ particularly inducible $\beta$-lactamase $(13,24)$. During the treatment by 3 rd generation of cephalosporins (3GS) and hyperproduction (high level cephalosporinase) of socalled derepressed AmpC $\beta$-lactamase, the therapy can fail (25).

The most notable carbapenemase production within Klebsiella spp. strain in Slovak republic is metallo- $\beta$-lactamase NDM (9) and this is in line with our findings, according to inhibition of MBL by EDTA in our assays.

Owing to the most prevalent sequence type - ST131, the outcomes of MacFadden et al (2019) about resistance within ST131 E. coli strains were higher than in susceptible strains, but different comparing to our overall E. coli resistance. In this Canadian re- search, resistance against ceftriaxone, ciprofloxacin and gentamicin ranged from $41-54 \%$ (26). Our susceptibility testing results of $E$. coli against $3 \mathrm{GS}$ and ciprofloxacin recorded 2-times higher values with exception of gentamicin with 2-times lower rate. Consistent with our data were Lafolie et al (2014) results from French hospital of 261 E. coli ESBL producers, in particular 45 ST131 strains were resistant to cefotaxime (93.3\%), ceftazidime (84.4\%), ofloxacin (91.1\%), amikacin (8.9\%) and carbapenems (0 \%) (21). Limitation of our resistance results in particular of ST131 E. coli strains was lower number of susceptibility results.

Antibiotic susceptibility profile of carbapenem resistant $K$. pneumoniae from long-term acute care hospitals from the work of Han et al (2017) was lower against amikacin (59.2\%) and mainly tigecycline $(0.7 \%)$, in line with ciprofloxacin $(98.1 \%)$, tobramycin $(97.8 \%)$, but higher than gentamicin $(97.8 \%)$ and especially than colistin (16.1\%) (20), as were our results. Differences in resistance rate can be explained by variety of their carbapenem resistant $K$. pneumoniae strains and our carbapenemase producing pathogens, other antibiotic pressure, distinction of geographical localisation and many other risk factors.

To our knowledge, empirical therapy of E. coli and Enterobacter spp. ESBL infections might comprise of non-carbapenem $\beta$-lactams: ceftazidime/avibactam, ceftolozane/tazobactam, amikacin, tigecycline, temocillin, nitrofurantoin and fosfomycin (27, 28 ). On the other hand, carbapenems are preferred initially for severely ill patients, if the source of possible bacteraemia is different than urinary or biliary tract, with high bacterial load and until final results become available (6). In terms of our data carbapenemase producing Klebsiella spp. is sensitive to colistin that is possible to combine with tigecycline in initial therapy. Efficacious in treatment of serine group A carbapenemase (KPC) Klebsiella spp. infection is for the time being ceftazidime/avibactam that demonstrated no resistance in our findings. For the therapy of MBL a few new coming agents like cefiderocol, aztreonam/avibactam, eravacycline and cefepime/zidebactam might be efficient $(29,30)$.

In conclusion, we have shown that multidrug-resistant, ESBL and CPE strain carriage and infections are related to various risk factors like age, hospitalization, healthcare setting, disease and many others. Mechanisms of resistance are most likely associated with particular infectious agent as well as sequence type, that might be highly specific and can have epidemic relationship and lead to nosocomial outbreak of infections. Consequently, prevalence of antimicrobial resistance is often in agreement with previous phenotypic and genotypic properties of pathogen, critical factors of the patients and surrounding hospital environment characteristics.

\section{References}

1. Karlowsky JA, Lob SH, Kazmierczak KM et al. In vitro activity of imipenem against carbapenemase-positive Enterobacteriaceae isolates collected by SMART global surveillance program from 2008 to 2014. J Clin Microbiol 2017; 55 (6): 1638-1649.

2. Barbier F, Pommier C, Essaied W et al. Colonization and infection with extended-spectrum $\beta$-lactamase-producing Enterobacteriaceae in ICU patients: what impact on outcomes and carbapenem exposure? J Antimicrob Chemother 2016; 71: 1088-1097. 
3. Olesen B, Hansen DS, Nilsson F et al. Prevalence and characteristics of the epidemic multiresistant Escherichia coli ST131 clonal group among extended-spectrum $\beta$-lactamase-producing E. coli isolates in Copenhagen, Denmark. J Clin Microbiol 2013; 51 (6):1779-1785.

4. Thomson KS. Extended-spectrum- $\beta$-lactamase, AmpC, and carbapenemase issues (commentary). J Clin Microbiol 2010; 48 (4): 1019-1025.

5. Gorrie CL, Mirceta M, Wick RR et al. Antimicrobial-resistant Klebsiella pneumoniae carriage and infection in specialized geriatric care wards linked to acquisition in the referring hospital. Clin Infect Dis 2018; 67 (2): 161-170.

6. Tamma PD, Rodríguez-Baño J. The use of noncarbapenem $\beta$-lactams for the treatment of extended-spectrum- $\beta$-lactamase infections. Clin Infect Dis 2017; 64 (7): 972-980.

7. Magiorakos AP, Srinivasan A, Carey RB et al. Multidrug-resistant, extensively drug-resistant and pandrug-resistant bacteria: an international expert proposal for interim standard definitions for acquired resistance. Clin Microbiol Infect 2012; 18: 268-281.

8. Cano A, Gutiérrez- Gutiérrez B, Machuca I et al. Risks of infection and mortality among patients colonized with Klebsiella pneumoniae carbapenemase-producing K. pneumoniae: validation of scores and proposal for management. Clin Infect Dis 2018; 66 (8): 1204-1210.

9. Logan LK, Weinstein RA. The epidemiology of carbapenem-resistant Enterobacteriaceae: the impact and evolution of a global menace. J Infect Dis 2017; 215 (S1): S28-S36.

10. Kluytmans-van den Bergh MF, Rossen JW, Bruijning-Verhagen PC et al. Whole-genome multilocus sequence typing of extended-spectrumbeta-lactamase-producing Enterobacteriaceae. J Clin Microbiol 2016; 54 (12): 2919-2927.

11. Huang TD, Bogaerts P, Berhin C, Guisset A, Glupczynski Y. Evaluation of brilliance ESBL agar, a novel chromogenic medium for detection of extended-spectrum-beta-lactamase-producing Enterobacteriaceae. J Clin Microbiol 2010; 48 (6): 2091-2096.

12. Giske CG, Martinez-Martinez L, Cantón R et al. EUCAST guidelines for detection of resistance mechanisms and specific resistances of clinical and/or epidemiological importance. The European Committee on Antimicrobial Susceptibility Testing 2017; version 2: 1-43. http://www. eucast.org/resistance_mechanisms/.

13. Tan TY, Yong Ng LS, He J, Koh TH, Hsu LY. Evaluation of screening methods to detect plasmid-mediated AmpC in Escherichia coli, Klebsiella pneumoniae, and Proteus mirabilis. Antimicrob Agents Chemother 2009; 53 (1): 146-149.

14. Nordman P, Poirel L, Dortet L. Rapid detection of carbapenemaseproducing Enterobacteriaceae. Emerg Infect Dis 2012; 18 (9): 1503-1507.

15. Poulou A, Grivakou E, Vrioni G et al. Modified CLSI extendedspectrum $\beta$-lactamase (ESBL) confirmatory test for phenotypic detection of ESBLs among Enterobacteriaceae producing various $\beta$-lactamases. J Clin Microbiol 2014; 52 (5): 1483-1489.

16. Ahmed S, Besser TE, Call DR, Weissman SJ, Jones LP, Davis MA. Evaluation of two multi-locus sequence typing schemes for commensal Escherichia coli from dairy cattle in Washington state. J Microbiol Methods 2016; 124: 57-61.
17. Roer L, Johannesen TB, Hansen $\mathbf{F}$ et al. CHtyper, a web tool for subtyping of extraintestinal pathogenic Escherichia coli based on the fumC and fimH alleles. J Clin Microbiol 2018; 56 (4): e00063-18. https://doi. org/10.1128/JCM.00063-18.

18. Gattringer R, Nikš M, Ostertág E et al. Evaluation of MIDITECH automated colorimeter MIC reading for antimicrobial susceptibility testing. J Antimicrob Chemother 2002; 49: 651-659.

19. http://www.eucast.org/clinical_breakpoints/

20. Han JH, Goldstein EJ, Wise J, Bilker WB, Tolomeo P, Lautenbach E. Epidemiology of carbapenem-resistant Klebsiella pneumoniae in a network of long-term acute care hospitals. Clin Infect Dis 2017; 64 (7): 839-844.

21. Lafolie J, Nicolas-Chanoine MH, Grenouillet F, Hocquet D, Bertrand X. Prevalence of Escherichia coli sequence type 131 and its H30 subclone among E. coli isolates in a french hospital. Int J Antimicrob Agents 2014; 44: 466-468.

22. Humphries RM, McKinnell JA. Continuing challenges for the clinical laboratory for detection of carbapenem-resistant Enterobacteriaceae. J Clin Microbiol 2015; 53 (12): 3712-3714.

23. Lesho E, Chukwuma U, Sparks $M$ et al. Anatomic, geographic, and taxon-specific relative risks of carbapenem resistance in the health care system of the U.S. department of defense. J Clin Microbiol 2016; 54 (6): 1546-1551.

24. Valsesia G, Roos M, Böttger EC, Hombach M. A statistical approach for determination of disk diffusion-based cutoff values for systematic characterization of wild-type and non-wild-type bacterial populations in antimicrobial susceptibility testing. J Clin Microbiol 2015; 53 (6): 1812-1822.

25. Jehl F, Chomarat M, Weber M, Gérard A (Eds). Resistance phenotypes of aerobic gram-negative bacteria to major antibiotics. 62-75. In: From antibiogram to prescription. Marcy L'étoile: Biomérieux, 2004.

26. MacFadden DR, Melano RG, Coburn B, Tijet N, Hanage WP, Daneman N. Comparing patient risk Factor-, sequence type-, and resistance locus identification-based approaches for predicting antibiotic resistance in Escherichia coli bloodstream infections. J Clin Microbiol 2019; 57 (6): e01780-18. https://doi.org/10.1128/JCM.01780-18.

27. Wijma RA, Huttner A, Koch BC, Mouton JW, Muller AE. Review of the pharmacokinetic properties of nitrofurantoin and nitroxoline. J Antimicrob Chemother 2018; 73: 2916-2926.

28. Vidyalakshmi PR, Ghafur KA, Gohel S, Thirunarayan MA. Fosfomycin, a promising option in the era of NDM1 susceptibility data with a discussion on its role in indian scenario. Infect Dis Clin Pract 2016; 24 (1): $35-38$.

29. Poulakou G, Lagou S, Karageorgopoulos DE, Dimopoulos G. New treatments of multidrug-resistant gram-negative ventilator-associated pneumonia. Ann Transl Med 2018; 6 (21). http://dx.doi.org/10.21037/ atm.2018.1029.

30. Sader HS, Castanheira M, Huband M, Jones RN, Flamm RK. WCK 5222 (cefepime-zidebactam) antimicrobial activity against clinical isolates of gram-negative bacteria collected worldwide in 2015. Antimicrob Agents Chemother 2017; 61 (5): e00072-17. https://doi.org/10.1128/ AAC.00072-17. 